\title{
¿Podría la Universidad Estatal a Distancia de Costa Rica establecer un modelo incubación regional?
}

\author{
Could the Distance State University of Costa Rica establish a regional \\ incubation model?
}

Doi: 10.22458/rna.v1112.2188

Dr. Jorge Camacho Barboza

Universidad Estatal a Distancia, Costa Rica

jcamachob@uned.ac.cr

https://orcid.org/0000-0002-7281-8405

\section{RESUMEN}

En muchos países del mundo existe la necesidad de impulsar y apoyar el emprendedurismo como alternativa para alcanzar el bienestar de sus pueblos. Al respecto, la Universidad Autónoma del Estado de Hidalgo (UAEH) ha promovido el fomento y apoyo a estudiantes y público en general para que generen sus propias empresas. Por otra parte, La Universidad Estatal a Distancia (UNED) ha jugado un papel importante en el apoyo del emprendimiento en las distintas regiones de Costa Rica. Una serie de buenas prácticas de la UAEH podrían ser adaptadas por la UNED para establecer un nuevo modelo de apoyo al emprendimiento.

\section{ABSTRACT}

In many countries around the world, the support and encouragement of entrepreneurship are seen as an alternative for the development of their communities. In this regard, the Autonomous University of Hidalgo State (UAEH) has promoted the support and development of students and people in general to create their own businesses. On the other hand, the Distance State University (UNED) has played an important role in supporting entrepreneurship in the various regions of Costa Rica. The UNED can adapt a series of best practices from the $\mathrm{UAEH}$ in order to define a new model to support entrepreneurship.

\section{RÉSUMÉ}

Dans de nombreux pays du monde se manifeste la nécessité d'impulser et de soutenir l'entreprenariat comme alternative pour parvenir au bien-être des populations. A Cet égard, l'Université Autonome de l'État d'Hidalgo (UAEH) a favorisé d'encourager et de soutenir les étudiants et le public en général afin qu'ils créent leurs propres entreprises. D’autre part, l'Université d'Etat à Distance (UNED) a joué un rôle important en donnant son appui à l'entreprenariat dans les diverses régions du Costa Rica. Une série de bonnes pratiques de l'UAEH pourraient être adaptées par l'UNED pour établir un nouveau modèle de soutien à l'entreprenariat.

\section{RESUMO}

Em muitos países do mundo existe a necessidade de impulsionar e apoiar o empreendedorismo como alternativa para alcançar o bem-estar de seus povos. Diante disto, a Universidad Autónoma del Estado de Hidalgo (UAEH) tem promovido a promoção e o apoio aos estudantes e ao público em geral, para que constituam suas próprias empresas. Por outro lado, a Universidad Estatal a Distancia (UNED) tem desempenhado um papel importante no apoio ao empreendedorismo nas diferentes regiões da Costa Rica. Uma série de boas práticas dos UAEH poderiam ser adaptadas pela UNED para estabelecer um novo modelo de apoio ao empreendedorismo.
PALABRAS CLAVE:

EMPRENDIMIENTO,

INCUBACIÓN,

REGIONAL,

DESARROLLO,

UNIVERSIDAD.
KEY WORDS:

ENTREPRENEURSHIP

INCUBATION,

REGIONAL,

DEVELOPMENT,

UNIVERSITY.
PALAVRAS CHAVE:

EMPREENDEDORISMO, INCUBAÇÃO, REGIONAL, DESENVOLVIMENTO, UNIVERSIDADE.
MOTS CLÉS:

ENTREPRENARIAT,

INCUBATION, REGIONAL,

DEVELOPPEMENT,

UNIVERSITE. 


\section{INTRODUCCIÓN}

Emprendimiento viene del término francés "entrepreneur" y se refiere principalmente al hecho de estar listos para tomar decisiones o iniciar algo nuevo. Esta palabra caracteriza a la persona emprendedora en un estado permanente de innovación, con alta motivación y compromiso por una tarea específica (Rodríguez, 2009). Los libros de administración la definen como la capacidad de iniciar y operar nuevas empresas con éxito (Mintzberg et al., 1999, como se citó en Rodríguez, 2009). Por otra parte, relacionado a este término, está el vocablo Incubadora el cual la Asociación Nacional de Entidades Promotoras de Emprendimientos Innovadores de Brasil lo define como una organización que promueve el desarrollo de emprendimientos con altos componentes de innovación. Una incubadora buscará guiar el proceso de desarrollo de ideas innovadoras a través de la facilitación de infraestructura y soporte gerencial, para así convertir dichas ideas en empresas exitosas (Bóbeda et al., 2015).

La Política de Fomento al Emprendimiento de Costa Rica (2014-2018), propuesta por su Ministerio de Economía, Industria y Comercio (MEIC), señala la existencia de varias fuerzas de cambio que llevan a plantear la necesidad de analizar la situación socio-económica y promover el emprendimiento para el crecimiento del país. Una de estas fuerzas plantea que las políticas de emprendimiento deben impulsar la creación de nuevos proyectos y empresas al alcance de cualquier persona, sin importar su situación socio-económica, esto con el objetivo de generar empleo y nuevos ingresos; la segunda fuerza evidencia que el emprendimiento social debe visualizarse para fomentar la implementación de proyectos que buscan resolver problemas sociales y ambientales que afectan a un gran número de personas y que generalmente deberían ser resueltos por el Estado.

Finalmente, hay otra fuerza que muestra como la innovación y las tecnologías han permitido la creación de nuevos productos y servicios en beneficio de emprendimiento (MEIC, 2014). Es importante señalar que para Audretsch y Keilbach; Aloulou y Aloulou; Wennekers, van Stel, Thurik y Reynolds (citados en Sánchez, Zerón y De La Garza, 2017) las naciones deben apoyar la creación de nuevas empresas con la finalidad de hacer frente a cambios en el entorno y mantener así su estabilidad macroeconómica.

En la Figura 1 se pueden observar a los actores del Ecosistema de Emprendimiento de Costa Rica agrupados por afinidad de la siguiente forma: Gobierno, Banca, Consejo Nacional de Emprendimiento, Red Nacional de Incubadores y Capacitadores, esto según lo indicado en la Política de Fomento al Emprendimiento de Costa Rica (2014-2018). En el primer grupo, Gobierno, se encuentra como ente rector del área el MEIC, adicionalmente se pueden mencionar a otros ministerios e institutos que son sus socios claves en el fomento del emprendimiento, entre dichos ministerios e institutos se encuentran: el Ministerio de Ciencia, Tecnología y Telecomunicaciones (MICITT), el Ministerio de Comercio Exterior (COMEX), la promotora de Comercio Exterior (Procomer), el Ministerio de Educación Pública (MEP), el Instituto Nacional de Aprendizaje (INA), el Instituto Nacional de la Mujer y el Instituto Mixto de Ayuda Social (IMAS) (MEIC, 2014).

El grupo Banca incluye a todas aquellas instituciones que cuentan con recursos financieros para apoyar el emprendimiento, tal es el caso del Sistema de Banca para el Desarrollo (SBD), del Banco Nacional de Costa Rica con el programa BN Desarrollo, el Banco Popular y Desarrollo Comunal como administrador del Fondo Especial para el Desarrollo de la Micros, Pequeñas y Medianas Empresas (BP FODEMIPYME) y las Microfinancieras (MEIC, 2014).

Por otra parte, el Consejo Nacional de Emprendimiento está integrado principalmente por instituciones públicas, privadas y la academia cuya función es promover, fomentar, coordinar, articular e implementar programas para fomentar el emprendimiento. Adicionalmente, existe la Red Nacional de Incubadoras, tanto privadas como de universidades públicas dónde se brinda apoyo a emprendedores con el objetivo de crear y propiciar nuevas oportunidades de negocios. Finalmente, hay un grupo importante de instituciones que forman, capacitan y educan en el tema del emprendimiento, entre dichas instituciones tenemos las universidades costarricenses y la Fundación Comisión Asesora en Alta Tecnología (CAATEC) (MEIC, 2014) 


\section{Figura 1}

Ecosistema de Emprendimiento de Costa Rica

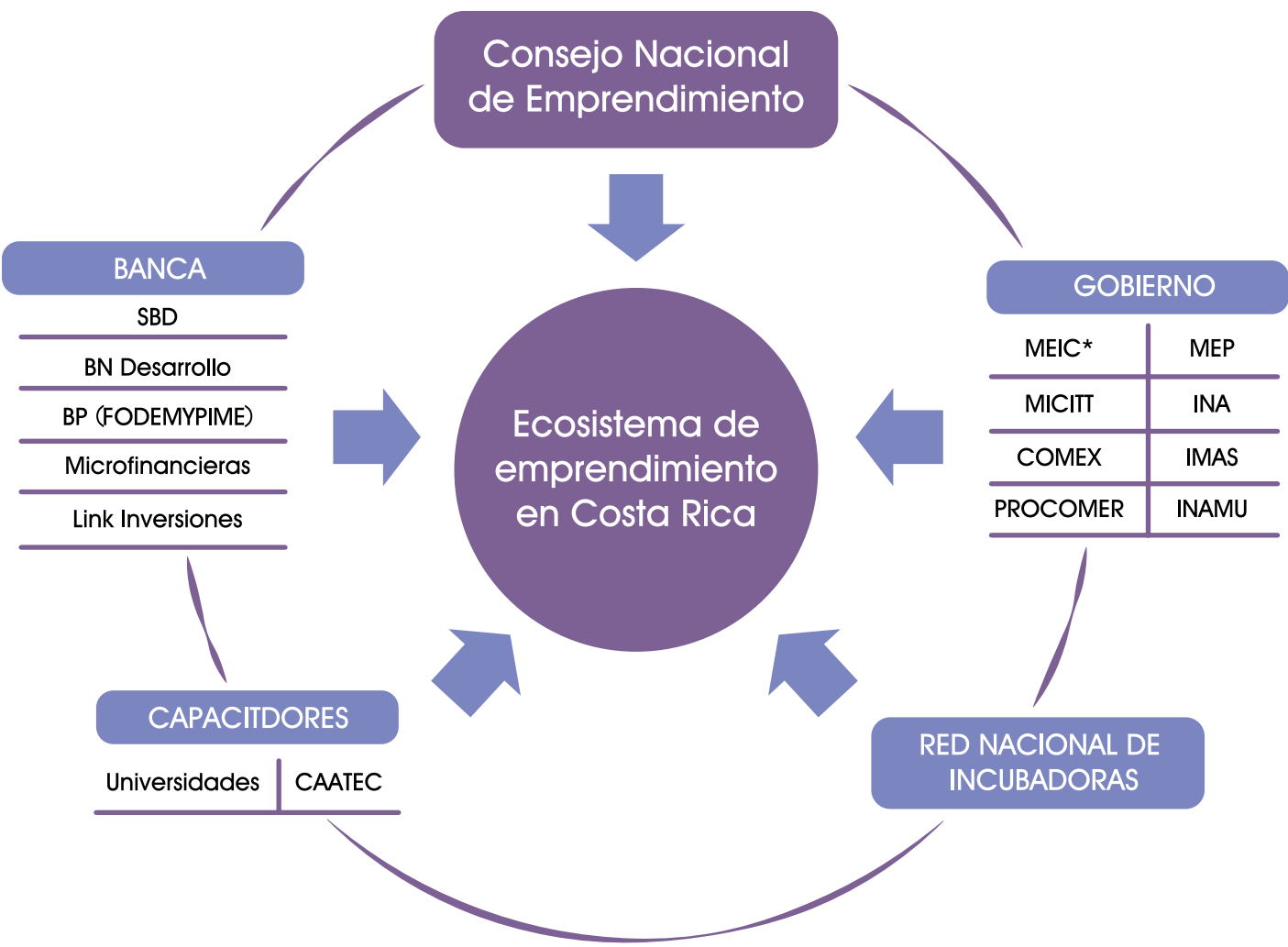

Fuente: elaboración propia con datos de la Política de Fomento al Emprendimiento de Costa Rica (2014-2018) del Ministerio de Economía, Industria y Comercio.

El Ministerio de Economía, Industria y Comercio (MEIC) y el Instituto Nacional de Aprendizaje(INA) en colaboración con las instituciones que forman parte de la Red de Apoyo a las Pequeñas y Medianas Empresas (PYME) en Costa Rica definen a una incubadora como la organización que acompaña el establecimiento de nuevas empresas, además, posee la infraestructura necesaria para facilitar dicho acompañamiento y una figura jurídica que norma su accionar (INA y MEIC, 2017); similar a esto, Peña-Vinces et al. (2011) definen a la incubadora como un programa que tiene como finalidad facilitar el surgimiento de emprendimientos tradicionales o de base tecnológica; en general, ofrecen los recursos necesarios para que dichos emprendimientos cumplan sus objetivos y logren convertirse en empresas sólidas y fuertes. Para Andrade Júnior (2012) el rol de una incubadora es realizar los esfuerzos necesarios para que pequeñas empresas tecnológicas, logren perdurar en el tiempo luego de su instalación inicial, brindando soporte en lo que se refiere a las acciones administrativas, tecnológicas y de comercialización.

Según la Estrategia Nacional de Empleo y Producción (2014-2018) del Gobierno de Costa Rica, en el 2012 el Parque Industria estuvo conformado por 48981 empresas de las cuales el 95\% correspondieron a PYME. El 77\% de la cuales pertenecen a los sectores del comercio, industria, servicio y tecnologías de información. Por otra parte, el 
comportamiento desagradado por regiones varía enormemente de la Región Central en donde se concentran el 81\% de las PYME, mientras que en las otras regiones del país la concentración PYME es muy baja, en la Región Caribe con un 3\% y un 4\% en cada una de las Regiones Brunca, Chorotega, Huetar Norte y Pacífico Central (Presidencia de la República de Costa Rica, MTSS y MEIC, 2014).

Por otra parte, en la Estrategia Nacional de Empleo y Producción (2014-2018) se indica que la creación y la calidad de empleo afecta principalmente a mujeres y jóvenes, así como a los territorios costeros y zonas rurales de menor desarrollo relativo. Por ejemplo, en el 2013 la tasa de desempleo de las mujeres fue de 10.8\%, en contraposición de la tasa para los hombres que fue de $7 \%$. En ese mismo año, el desempleo para los más jóvenes (15 a 25 años) fue de $18.5 \%$, mientras que para otro grupo de más edad (26-35 años) fue de 8.4\%. Adicionalmente, también el 2013, las regiones Caribe, Chorotega y Brunca presentaron tasas de desempleo de cerca de $11 \%$, mientras que la región Central presentó una tasa de 7.7\% (Presidencia de la República de Costa Rica, MTSS y MEIC, 2014).

Como se detallará más adelante, la Universidad Estatal a Distancia (UNED) de Costa Rica ha incursionado en múltiples iniciativas de apoyo al emprendimiento; sin embargo, no cuenta con un modelo de incubación UNED. Por otra parte, dentro de la coyuntura nacional descrita anteriormente, se plantea la necesidad de que la UNED conozca que hacen otras universidades para fomentar el emprendimiento y la incubación. Por tal motivo, el encargado de la Unidad de Enlace Universidad Sector Externa de la Dirección de Internacionalización Cooperación de la UNED realizó una pasantía a la Universidad Autónoma del Estado de Hidalgo (UAEH) con el propósito de conocer el sistema de incubación de dicha institución el cual tiene el objetivo de obtener información relevante para el establecimiento de un modelo de incubación y dinamización del emprendimiento en la UNED.

\section{Figura 2}

Fases de Pre-Incubación, Incubación y Post-Incubación del Modelo de Incubación del Centro Incubador de Empresas de la Universidad Autónoma del Estado de Hidalgo

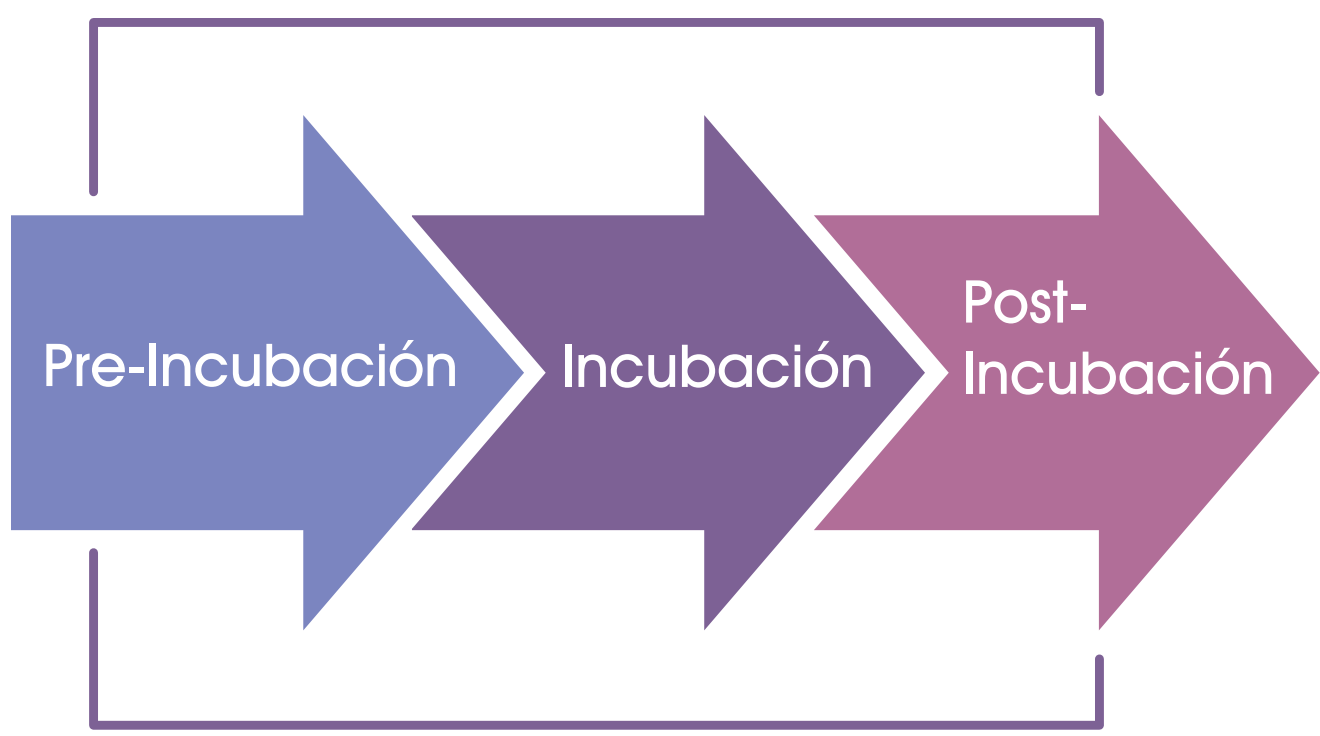

Fuente: elaboración propia con datos de la Política de Fomento al Emprendimiento de Costa Rica (2014-2018) del Ministerio de Economía, Industria y Comercio. 
Los proyectos en la segunda fase de Incubación entran en un proceso donde se le brinda el soporte necesario para aplicar el plan de negocios e iniciar las operaciones de la nueva empresa. Es importante mencionar que los proyectos a los cuales se les asesore para optar por recursos financieros, que provienen de concursos a distintos fondos del Estado o de organismos que financian el sector, firman un contrato para reintegrar a la UAEH $5 \%$ de los fondos obtenidos (Rabling D., comunicación personal, 20 de junio de 2016).

Finalmente, la tercera fase de Post-Incubación brinda acompañamiento a las empresas incubadas con el objetivo de garantizar la consolidación de estas a lo largo del tiempo, en esta fase se promueve la actualización del plan de negocios y el intercambio de experiencias con emprendedores exitosos. Esta última fase tendrá un costo de un $3 \%$ de las ganancias anuales de la empresa una vez alcanzado el punto de equilibrio, dicho dinero ingresa a la universidad como donación y se puede deducir de los impuestos que debe pagar la empresa incubada al gobierno (Rabling D., comunicación personal, 20 de junio de 2016).

Para implementar el Modelo Institucional de Incubación (Figura 2), el CIE cuenta con una Incubadora de Base Intermedia que opera en el Campus Central de la UAEH en la Cuidad de Pachuca, este modelo utilizado en el Campus Central ha sido replicado a ocho Escuelas Superiores (sedes regionales) de la UAEH; así como a los Institutos de Artes y de Ciencias Agropecuarias de la Universidad y a la Fundación Hidalguense para personas con capacidades diferentes, esto ha permitido potenciar el número de solicitud de asesorías para incubar empresas. Estas réplicas o clones de la Incubadora de Base Intermedia cuentan con 1 o 2 funcionarios por sede, los cuáles extra a sus funciones, son capacitados por el CIE en todo lo referente a los procesos del Modelo Institucional de Incubación, esto con el objetivo de que puedan liderar en las Escuelas Superiores e Institutos el proceso de incubación de nuevas empresas (Rabling D., comunicación personal, 20 de junio de 2016).

Por otra parte, el Centro Incubador de Empresas realiza tres talleres al año para buscar proyectos emprendedores productivos en donde se capacitan en elaboración de plan de negocios cerca de 90 proyectos. En las Escuelas Superiores e Institutos se realiza un taller por año en el cual se capacitan en promedio 10 proyectos, por tanto, se llegan a asesorar cerca de 200 proyectos por año en el estado de Hidalgo. Adicionalmente, el proceso de incubación de empresas dentro de la UAEH ha obtenido gran relevancia, debido a que el Gobierno del Estado de Hidalgo ha establecido este proceso como requisito para aplicar a fondos del Estado para apoyar el emprendimiento, obligando al emprendedor a sumarse al proceso de incubación (Rabling D., comunicación personal, 20 de junio de 2016).

Dentro de la estrategia para promover el proceso de incubación, tanto en el Centro Incubador de Empresas como en las Escuelas Superiores e Institutos de la Universidad, se utilizan una serie de recursos para alcanzar sus objetivos, primero es importante mencionar que dichas actividades se hacen con el apoyo de estudiantes, principalmente aquellos que hacen sus tesis o su servicio social, requisitos que deben cumplir en sus programas de estudio. Cómo segunda práctica a los consultores que participan en los talleres o dan acompañamiento en la incubación de los proyectos, se les paga con recursos que obtiene el Centro Incubador; sin embargo, cabe mencionar que en algunos casos su colaboración es voluntaria y muchos profesionales jóvenes la utilizan como plataforma para adquirir experiencia en sus áreas profesionales (Rabling D., comunicación personal, 20 de junio de 2016).

El Centro Incubador de Empresas busca también fortalecer la cultura en materia de Propiedad Intelectual en emprendedores, alumnos e investigadores de la Universidad, de las Escuelas Superior (sedes regionales) e Institutos. Para lograr este objetivo se cuenta con una persona encargada de tópicos tales como: propiedad industrial y registro de marcas, quién como parte del proceso de incubación asesora sobre las gestiones necesarias para garantizar la protección de los derechos de autor y el registro de marcas de los proyectos incubados (Reyes L.L., comunicación personal, 20 de junio de 2016).

\section{Propuesta Incubadora UNED}

La Universidad Estatal a Distancia (UNED) inicia sus actividades bajo el amparo de la Ley de Creación No. 6044 aprobada por la Asamblea Legislativa de Costa Rica y pública en el periódico oficial la Gaceta el 12 de marzo de 1977. Su misión es ofrecer educación a todos los sectores de la población, especialmente a aquellos que por razones económicas, sociales, geográficas, culturales, etarias, de discapacidad o de género requieren oportunidades para una inserción real y equitativa en la sociedad. 
La UNED ha desarrollado su propio modelo pedagógico de educación a distancia, con el cual busca alcanzar su misión, en él se concretiza el proceso de aprender y ensañar a distancia, precisando y orientando la estructuración y presentación de los contenidos de formación, la facilitación y evaluación de los aprendizajes, así como las formas de apoyo y servicio que hagan posible la existencia y funcionamiento de dicho modelo (Consejo Universitario UNED, 2005). Complementariamente a esto, siempre con el objetivo de alcanzar su misión, la UNED se ha extendido por todo el territorio costarricense a través de sus 36 Centros Universitarios o sedes (Figura 3), así como con la utilización de una plataforma en línea y de recursos tecnológicos para materializar dicho modelo. La infraestructura y el modelo pedagógico en la UNED son recursos claves con los que cuenta la institución para llevar a cabo capacitación y asesoramiento a emprendimientos en las distintas regiones del país.

Figura 3

Distribución de los Centros Universitarios de la Universidad Estatal a Distancia

a lo Largo de Todo el Territorio Nacional en Costa Rica.

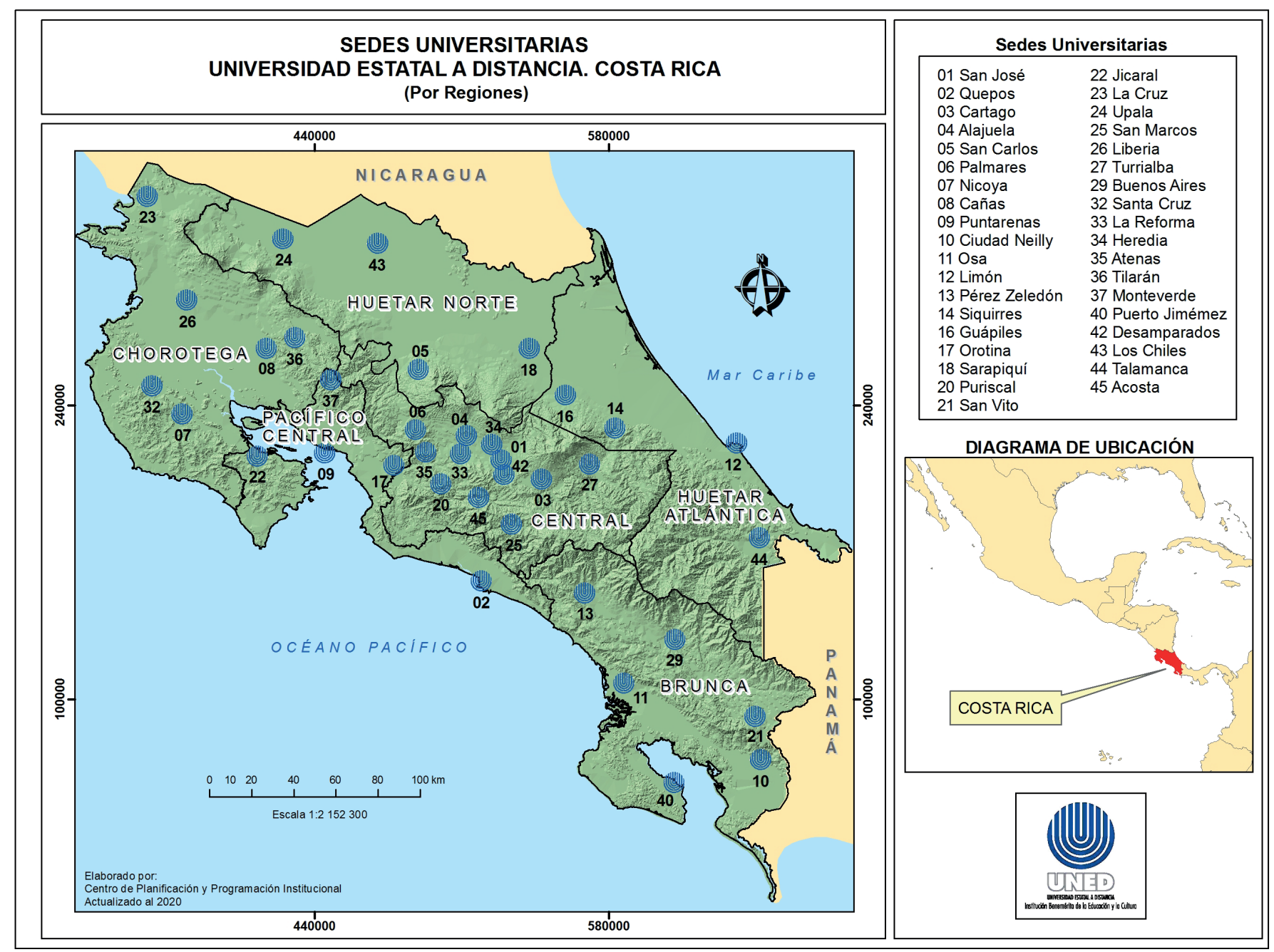

Fuente: UNED Costa Rica. 
En materia de emprededurismo, la UNED cuenta con amplia experiencia relacionada con la capacitación y el fomento de emprendimientos, en el 2005 el Observatorio de MIPYMES nace para llevar información y conocimiento sobre la gestión empresarial a las micro, pequeñas y medianas empresas (MIPYMES). Otros ejemplos de ello son proyectos tales como Empléate, una iniciativa del Ministerio de Trabajo y Seguridad Social (MTSS) con apoyo de instituciones públicas como la UNED en dónde se facilitaron procesos educativos para impulsar y promover el empleo de los jóvenes de las distintas regiones del país, en particular, la Escuela de Ciencias en Administración (ECA) de la UNED impartió el curso "Emprendedurismo" que tuvo como objetivo promover el desarrollo empresarial y facilitar de herramientas a pequeños y medianos empresarios (Kcuno, 2015).

Emprende Rural es otro proyecto que ha impulsado la UNED, con éste se busca el fomento del emprendedurismo y el desarrollo micro-empresarial en el espacio rural, este proyecto busca a mediano plazo el desarrollo e implementación de una estrategia educativa orientada a favorecer en las personas el desarrollo integral, la empleabilidad y la generación de ingresos, así como instalar un consultorio empresarial para el micro-empresario que desee consultar un tema del que requiere acción inmediata para el mejoramiento de su microempresa (Ramírez, 2016).

El Programa de Desarrollo Gerencial de la Dirección de Extensión, la Escuela de Ciencias en Administración (ECA) y la Escuela de Ciencias Exactas y Naturales (ECEN) son las dependencias académicas dentro de la UNED, con mayor experiencia y trayectoria en capacitación, asesoría y fomento del emprendimiento dentro de la institución. En los últimos años la ECA ha organizado un evento al año llamado "Jornadas Emprededoras" que busca desarrollar ambientes amigables para impulsar ideas innovadoras de emprendedurismo (Kcuno, 2017); recientemente, la ECEN incorpora dentro de su quehacer el desarrollo de capacitaciones en el tema del emprendimiento, esto mediante la organización del primer Seminario de Actualización Profesional de la carrera de Manejo de Recursos Naturales en el Centro Universitario de Nicoya el cual tuvo entre sus objetivos desarrollar actividades prácticas y teóricas en emprendedurismo, aplicados al manejo de recursos naturales y su integración con la actualización profesional de los estudiantes (Kcuno, 2017).

En esta misma línea, la ECEN también organizó en mayo del 2018 el primer Encuentro de Agroemprendedores que buscó abrir un espacio para que estudiantes, tutores, graduados y productores expusieran sus experiencias emprendedoras (Ramírez, 2018).

A mediados del 2015 se establece la Red UNED Emprende que busca la articulación institucional de todas las iniciativas en materia de emprendimiento en la UNED (Ramírez, 2015). Dicha Red tiene como objetivo potenciar los emprendimientos e innovación social desde la Universidad Estatal a Distancia, con una perspectiva creativa, responsable y ambientalmente sustentable que favorezca a la mayor cantidad de personas y comunidades, así como la generación de conocimiento. Finalmente, en el 2016 con el establecimiento del Centro de Apoyo a la Tecnología y la Innovación (CATI) en la Dirección de Internacionalización y Cooperación de la UNED, se complementa la capacitación a emprendedores en materia de propiedad intelectual, esto mediante la promoción de información sobre la creación, protección, titularidad y gestión de los derechos propiedad intelectual y el fomento del emprendedurismo local a través de la asesoría para el acceso a información legal y técnica en materia de nuevas tecnologías.

Todas las experiencias presentadas anteriormente muestran una serie de esfuerzos aislados en materia de emprendimiento los cuales deben articularse y sistematizarse para lograr un mayor impacto sobre la población estudiantil y la población en general de las regiones en donde tiene presencia la UNED y en donde ha incursionado en apoyo al emprendimiento.

\section{CONCLUSIONES}

En la Política de Fomento al Emprendimiento de Costa Rica (2014-2018) se señaló la necesidad de analizar la situación socio-económica y promover el emprendimiento para el crecimiento del país. Esto complementado con la información que brinda la Estrategia Nacional de Empleo y Producción (2014-2018), en la cual se indica que las Regiones Caribe, Brunca, Chorotega, Huetar Norte y Pacífico Central son la regiones en dónde hay una menor concentración de PYME y el mayor desempleo del país; además de lo establecido en la misión de la UNED, que indica que la institución promoverá en las poblaciones más vulnerables una inserción real y equitativa en la sociedad; no obstante, hacen evidente la fortaleza que tiene la UNED en conocimiento, presencia en todo el país, infraestructura 
y recursos para valorar la factibilidad de desarrollar y probar un Modelo Incubador Regional en la UNED.

A partir del análisis anterior se plantea la necesidad de diseñar, en forma colaborativa con los expertos y actores interesados en el tema de emprendimiento, una propuesta del proceso de incubación para la UNED la cual deberá ir de la mano con la misión y visión de la Universidad.

El modelo de incubación regional que se proponga deberá tomar en cuenta las siguientes consideraciones:

- Desarrollar el modelo de incubación de acuerdo con las particularidades del sector empresarial de cada región, así como la definición de la malla curricular respectiva para capacitar a los emprendedores y la determinación de los recursos académicos, administrativos y financieros con que se contaría y los que habría que buscar.

- Establecer alianzas con socios regionales o nacionales, para obtener recursos con el fin de financiar los procesos de incubación, tales como podrían ser la Junta de Desarrollo Regional de la Zona Sur (Judesur), Instituto de Desarrollo Rural (Inder), el Sistema de Banca para el Desarrollo, los Gobiernos Locales, entre otros.

- Los estudiantes que deben realizar sus Trabajos Finales de Graduación podrían ser un recurso muy valioso para facilitar los procesos de incubación.

- La Fundación de la Universidad Estatal a Distancia para el desarrollo y promoción de la educación a distancia (FUNDEPREDI) podría utilizarse para la gestión de los recursos necesarios en el proceso de incubación.

- El modelo incubador se podría iniciar en alguna de las regiones donde la UNED tiene presencia, estas regiones son: Central, Brunca, Huetar Atlántica, Huetar Central, Chorotega y Pacífico Central, definiendo en cuál de los centros universitarios que conforman cada región, sería más estratégico que esté la incubadora regional. Esto sería una prueba piloto para poner en funcionamiento la incubadora. Dependiendo de su éxito, el modelo de incubación podría clonarse a las otras regiones del país.

- Compromiso de la academia y la administración de asignar tiempo a sus docentes para asesoría técnica a los proyectos.

- El Centro de Apoyo a la Tecnología y la Innovación (CATI) de la Dirección de Internacionalización y Cooperación de la UNED apoyaría a la incubadora en materia de propiedad intelectual, específicamente en las áreas de registros de marcas, modelos de utilidad, diseños industriales, patentes y secretos industriales. 


\section{REFERENCIAS}

Andrade Júnior (2012). La Experiencia Brasileña en la Superación de Dificultades de Empresas de Base Tecnológica en Incubadora. J. Technol. Manag. Innov., 7(3), 161-171.

Bolaños, R. (2017). La Investigación Cualitativa en las Ciencias de la Administración: Aproximaciones Teórico-Metodológicas. Revista Nacional de Administración, 8 (1), 25-45.

Bóveda, J.E., Oviedo, A., Yakusik, A.L. (2015). Manual de Implementación de Incubadoras de Empresas [Archivo PDF] https://www.jica.go.jp/paraguay/espanol/office/others/c8hovmooooad5gke-att/info_11_02.pdf

Consejo Universitario UNED (2005). Modelo pedagógico. [Archivo PDF] https://www.uned.ac.cr/academica/images/ igesca/materiales/24.pdf

Instituto Nacional de Aprendizaje y Ministerio de Economía, Industria y Comercio (7 de setiembre de 2017). PYMEs Costa Rica Ventana Única PYME. http://www.pyme.go.cr/cuadro6.php?id=1

Kawilich, Barbara B. (2005). La observación participante como método de recolección de datos. Forum: Qualitative Social Research Sozialforschung, 6(2), Art. 43. http://www.qualitative-research.net/index.php/fqs/article/ view/466/998\#g2

Kcuno, R. (18 de setiembre de 2017). UNED promueve emprendedurismo estudiantil. Acontecer. https://www.uned.ac.cr/ acontecer/a-diario/gestion-universitaria/2995-uned-promueve-emprendedurismo-estudiantil

Kcuno, R. (19 de junio 2015). Cientos de Jóvenes llegaron al Reto Empléate. Acontecer. https://www.uned.ac.cr/acontecer/ a-diario/gestion-universitaria/2310-cientos-de-jovenes-llegaron-al-reto-empleate

Kcuno, R. (21 de noviembre de 2017). Programa de Manejo de Recursos Naturales realizó "I Seminario de Actualización Profesional”. Acontecer. https://www.uned.ac.cr/acontecer/a-diario/gestion-universitaria/3076-programa-demanejo-de-recursos-naturales-realizo-i-seminario-de-actualizacion-profesional

Ministerio de Economía, Industria y Comercio. (2014). Política de Fomento al Emprendimiento de Costa Rica. [Archivo PDF].

http://www.pyme.go.cr/media/archivo/normativas/politicafomentoalemprendimiento.pdf

Peña-Vinces, J.C., Bravo, S., Álvarez, F.A. y Pineda, D.A. (2011). Analysis of Characteristics of Business Incubators in Colombia: A Case Study. J. econ. finance adm. sci., 16(30), 13-29.

Presidencia de la República de Costa Rica, Ministerio de Trabajo y Seguridad Social y Ministerio de Economía, Industria y Comercio. (2014). Estrategia Nacional de Empleo y Producción. [Archivo PDF]. http://www.mtss.go.cr/ elmininisterio/despacho/Estrategia\%20Nacional\%20de\%20Empleo\%20y\%20Produccion.pdf

Ramírez, K. (06 de julio de 2015). UNED Emprende: la nueva red institucional. Acontecer. https://www.uned.ac.cr/ acontecer/a-diario/gestion-universitaria/2320-uned-emprende-la-nueva-red-institucional

Ramírez, K. (22 de marzo de 2018). UNED celebrará I Feria de Agroemprendedores en mayo. Acontecer. https:// www.uned.ac.cr/acontecer/a-diario/juncos/51-a-diario-/gestion-universitaria/3145-uned-celebrara-i-feria-deagroemprendedores-en-mayo

Ramírez, K. (29 de febrero de 2016). Emprende Rural...más cerca de la micro, pequeña y mediana empresa. Acontecer. https:// www.uned.ac.cr/acontecer/a-diario/gestion-universitaria/2566-emprende-rural-mas-cerca-de-la-micro-pequena-ymediana-empresa

Rodríguez Ramírez, A. (2009). Nuevas perspectivas para entender el emprendimiento empresarial. Pensam. Gest. 26, 94-1 19. http://rcientificas.uninorte.edu.co/index.php/pensamiento/issue/view/59

Sánchez M.L., Zerón M. y De La Garza M.H. (2017). Intención emprendedora en estudiantes universitarios en el centro de Tamaulipas, México. Revista Nacional de Administración, 8 (2), 35-49.

Universidad Autónoma del Estado de Hidalgo (7 de setiembre de 2017). Estado de Hidalgo, México. https://www.uaeh.edu. $\mathrm{mx} /$ noticias/2644/ 